\title{
Konkurrierende Tarifpolitik: Herausforderung für die DGB-Gewerkschaften
}

Die Tarifgeschichte der Bundesrepublik ist von den DGB-Gewerkschaften geprägt, aber nie vollständig monopolisiert worden. Tarifpolitische Konkurrenz zu anderen Gewerkschaften war stets vorhanden; ihre Formen und ihre Dimension haben sich jedoch seit Ende der 1990er Jahre erheblich verändert. Eine nähere Analyse zeigt, dass unter gewerkschaftspolitischen Gesichtspunkten nicht die eigenständige Tarifpolitik der Berufsgewerkschaften, sondern die Unterbietungskonkurrenz durch die Mitgliedsverbände des christlichen Gewerkschaftsbundes (CGB-Gewerkschaften) das eigentliche Problem darstellt: Unterbietung wird durch Politik und Arbeitgeber gefördert. Tarifpolitische Kooperation ist hier kaum vorstellbar.

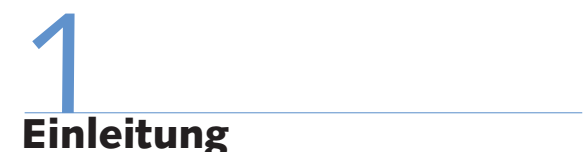

\section{Einleitung}

Gewerkschaften konkurrieren in Deutschland im Wesentlichen auf drei miteinander verschränkten Feldern. Das erste ist der Wettbewerb um Mitglieder, der überall dort stattfindet, wo sich Organisationsbereiche überschneiden. Als zweites Feld lässt sich die Gremienkonkurrenz benennen, bei der um Betriebs- und Aufsichtsratsmandate gestritten wird. Der dritte Kernbereich zwischengewerkschaftlichen Wettbewerbs ist schließlich die Tarifpolitik. Unter tarifpolitischer Konkurrenz wird im Folgenden der Versuch verstanden, in Abgrenzung zu einer anderen Gewerkschaft eigenständige Tarifverträge möglichst exklusiv in den selbst beanspruchten Geltungsbereichen durchzusetzen.

Im Fokus dieses Beitrags steht die Tarifpolitik. Zunächst werden die unterschiedlichen Formen tarifpolitischer Konkurrenz definiert (Abschnitt 2), dann wird ihr historisches Auftreten in den letzten Jahrzehnten dargestellt (Abschnitt 3). Das Ausmaß der Konkurrenz, das heißt ein Überblick über die konkurrierende Organisationslandschaft und die Beschäftigtengruppen, für die konkurrierende Tarifverträge vorliegen, sind Gegenstand des vierten Abschnitts. Basierend auf den dargestellten Entwicklungen werden gewerkschaftspolitische Konsequenzen diskutiert (Abschnitt 5). Der Beitrag schließt mit einem Ausblick auf die strategischen Positionen von Gewerkschaften und Arbeitgebern (Abschnitt 6).

\section{2}

\section{Formen tarifpolitischer Konkurrenz}

In der Tarifpolitik können idealtypisch zwei Formen konkurrierenden Gewerkschaftshandelns unterschieden werden, die sich als Überbietungs- und Unterbietungskonkurrenz kennzeichnen lassen (Zeuner 2007; Bispinck/Dribbusch 2008; Schroeder 2008). Beide heben sich nicht nur in der Zielrichtung ihrer Tarifpolitik voneinander $a b$, sondern bedingen auch unterschiedliche Machtkonstellationen und differieren deshalb in ihren Voraussetzungen und ihrer Durchsetzung.

Tarifpolitischer Wettbewerb folgt nicht zwangsläufig aus der Konkurrenz um Mitglieder oder Mandate, wofür zahlreiche Beispiele tarifpolitischer Kooperation zwischen ansonsten konkurrierenden Gewerkschaften stehen. In vielen Branchen und Betrieben sind zudem die gewerkschaftlichen Macht- und Einflusssphären und damit die Tarifführerschaften so eindeutig geklärt, dass es kaum Spielräume für konkurrierende Tarifabschlüsse gibt. Zwar ist auch das Binnenverhältnis innerhalb des Deutschen Gewerkschaftsbundes (DGB) keineswegs frei von Spannungen, die Hauptkonflikte treten aber im Verhältnis zwischen den DGB-Gewerkschaften und ihren Konkurrentinnen auf.

Tarifpolitik wird vor dem Hintergrund der jeweiligen institutionellen und politischen Rahmenbedingungen vor allem durch spezifische, veränderbare und sich wandelnde Kräfteverhältnisse zwischen Unternehmen und Gewerkschaften geprägt. Ein Schlüsselfaktor ist die gewerkschaftliche Durchsetzungs- und Streik- fähigkeit. Diese speist sich im Wesentlichen aus zwei miteinander verschränkten Quellen (Silver 2005): zum Ersten aus struktureller Verhandlungsmacht, die auf günstigen ökonomischen Rahmenbedingungen wie beispielsweise vollen Auftragsbüchern oder auch auf einer strategisch wichtigen Stellung der Beschäftigten im Produktionsoder Verwaltungsablauf basiert; zum Zweiten aus Mitgliederstärke und damit verbundener Mobilisierungsfähigkeit. Das fundamentale Unterscheidungsmerkmal beider Formen tariflicher Konkurrenz ist, dass Überbietungskonkurrenz sowohl strukturelle wie organisatorische Macht voraussetzt, während Unterbietungskonkurrenz gänzlich ohne jegliche Machtressource auskommt. Dies hat entscheidend mit der zugrundeliegenden Zielsetzung zu tun.

Als Überbietungskonkurrenz lässt sich eine Tarifpolitik bezeichnen, in der die Konkurrenzorganisation mit dem Anspruch antritt, durch eigenständiges Verhandeln bessere Tarifergebnisse erzielen zu können als die bisherige Tarifführerin. Dieses Anliegen stößt nicht nur auf die Ablehnung der bisher tarifführenden Gewerkschaft, sondern insbesondere auch auf den Widerstand der betreffenden Unternehmen und Arbeitgeberverbände. Letztere fürchten, dass ein Erfolg dieser Gewerkschaften ihre Personalkosten in die Höhe treibt und zukünftige Tarifverhandlungen durch das Auftreten konkurrierender Gewerkschaften komplizierter und konfliktreicher werden. Derartige Tarifverträge las-

Heiner Dribbusch, Dr., Wissenschaftler im WSI in der Hans-Böckler-Stiftung. Arbeitsschwerpunkte: Industrielle Beziehungen, Arbeitskampfentwicklung. e-mail: Heiner-Dribbusch@boeckler.de 
sen sich deshalb nur im Konflikt durchsetzen und erfordern entsprechende Durchsetzungskraft. Nicht zufällig handelt es sich bisher in allen Fällen erfolgreicher Überbietungskonkurrenz um Berufsgewerkschaften, die relativ homogene Beschäftigtengruppen vertreten, die über substanzielle strukturelle Macht verfügen und ausgezeichnet organisiert sind.

Im Gegensatz dazu findet sich die Unterbietungskonkurrenz überwiegend in Branchen, in denen die Beschäftigten über wenig strukturelle Macht verfügen und gering organisiert sind. Die unterbietende Gewerkschaft benötigt auch keinerlei Arbeitskampffähigkeit, da ihre Tarifübereinkünfte entweder die direkte Unterschreitung bestehender Tarifnormen oder die Zustimmung zu Regelungsinhalten beinhalten, zu deren Abschluss die bisher tarifschließende Gewerkschaft nicht bereit ist. Das von den Unternehmen zu kalkulierende Risiko besteht im Widerstand der Beschäftigten und der ausgebooteten Gewerkschaft. Entsprechend lautet ein „Praxistipp " für Unternehmen in der arbeitgebernahen Zeitschrift Arbeit und Arbeitsrecht: „Der Abschluss eines CGM-Firmentarifvertrages zur "Verdrängung“ eines DGBFlächentarifs kann dann empfehlenswert sein, wenn der DGB-Organisationsgrad der Belegschaft gering ist [...] und auch bei dessen Durchführung nicht mit massiven Neueintritten in die DGB-Gewerkschaft zu rechnen ist" (Wilhelm/Dannhorn 2006, S. 344).

\section{3}

In der Entwicklung der Tarifkonkurrenz lassen sich zwei Phasen unterscheiden: Die erste Phase etablierter und institutionell eingebetteter Konkurrenz um Tarifführerschaft wurde Mitte der 1990er Jahre von einer Phase neuen, radikalisierten Tarifwettbewerbs abgelöst.

\subsection{STREIT UM TARIFFÜHRERSCHAFT}

Zwischen der Deutschen Angestelltengewerkschaft (DAG) und den DGB-Gewerkschaften bestand über viele Jahrzehnte eine zeitweise geradezu feindselige Konkurrenz, die sich auch auf die Tarifpolitik erstreckte. Diese betraf in starkem Maße das Bankenund Versicherungsgewerbe wie auch den
Einzelhandel, wo die DAG auf die Gewerkschaft Handel, Banken, Versicherungen (HBV) traf (Bayer 1980). Beide Gewerkschaften stritten um die Tarifführerschaft. Verhandelt wurde jeweils getrennt mit den Arbeitgeberverbänden, doch zumeist kamen die Tarifparteien zu identischen Ergebnissen, die in Form separater Tarifverträge niedergelegt wurden. Ähnliche Verhältnisse bestanden im öffentlichen Dienst. Hier agierten neben der tarifführenden Gewerkschaft Öffentliche Dienste, Transport und Verkehr (ÖTV) und den anderen im öffentlichen Dienst vertretenen DGB-Gewerkschaften die DAG, der Marburger Bund (MB) sowie ab den 1970er Jahren eine aus Gewerkschaften des Deutschen Beamtenbundes ( $\mathrm{dbb}$ ) gebildete Gemeinschaft von Gewerkschaften und Verbänden des öffentlichen Dienstes (GGVöD) (u. a. Keller 1993, S. 153-159).

Eine weit zurückreichende Gewerkschaftskonkurrenz gibt es bei den Journalisten. Der 1949 gegründete Deutsche Journalistenverband (DJV), der nicht dem DGB beitrat, dominierte hier bis in die 1960er Jahre die Tarifpolitik. Nachdem jedoch Ende der 1960er Jahre die zur IG Druck und Papier gehörende Deutsche Journalisten Union (dju) gegenüber den Verlegern ihre Anerkennung als eigenständige Tarifpartei durchsetzen konnte, ergab sich bald ein gemeinsames Vorgehen bei Tarifverhandlungen, da aufgrund eines bis heute andauernden relativen Patts zwischen beiden Gewerkschaften keine ohne die andere befriedigende Ergebnisse erzielen konnte.

Der Christliche Gewerkschaftsbund Deutschlands (CGB), 1959 in bewusster Abgrenzung zum DGB gegründet, spielte bis in die 1990er Jahre tarifpolitisch keine nennenswerte Rolle. Aufgrund ihrer ausgeprägten Mitgliederschwäche blieben die CGB-Gewerkschaften weitestgehend darauf angewiesen, dass Arbeitgeberverbände die zuvor von den DGB-Gewerkschaften abgeschlossenen Tarifvereinbarungen mit ihnen in Form von Anschlusstarifverträgen noch einmal unterzeichneten.

\subsection{RADIKALISIERTE TARIFKONKURRENZ}

Die 1990er Jahre stellen in mehrerer Hinsicht einen Wendepunkt in der bundesdeutschen Tarifgeschichte dar. Die wirtschaftlichen Umwälzungen in Ostdeutschland und eine scharfe Rezession verliefen parallel zu einer sich beschleunigenden In- ternationalisierung der Wirtschaft und verstärkter Standortkonkurrenz. Ausdruck eines veränderten Wettbewerbsdrucks, aber auch eines wachsenden Selbstvertrauens der Unternehmen, war die in der zweiten Hälfte der 1990er Jahre von Arbeitgeberseite eingeleitete "tarifpolitische Wende“. Das alte sozialpartnerschaftliche Arrangement der Nachkriegszeit einschließlich des traditionellen Flächentarifvertragssystems wurde infrage gestellt (Bispinck 2006). Auf der betrieblichen Ebene folgten laufend neue Kostensenkungsprogramme, Ausgliederungen, Stilllegungen und Verlagerungen oder diesbezügliche Drohungen. In der politischen Arena setzten sich neoliberale Politikansätze durch. Erhöhung des Arbeitszwanges und Deregulierung der Beschäftigungsverhältnisse waren Elemente einer politisch gewollten Ausdehnung des Niedriglohnsektors. Der öffentliche Sektor - lange Zeit eine Bastion sozialstaatlich regulierter, nicht prekärer Lohnarbeit - wurde in großem Stil privatisiert und dereguliert (Dribbusch/Schulten 2007). Zusammen mit anhaltender Massenarbeitslosigkeit bildeten diese Entwicklungen die Rahmenbedingungen für neue Konstellationen von Tarifkooperation und Tarifkonkurrenz.

\section{NEUE KOOPERATIONEN}

Arbeitsplatzabbau in den traditionellen Industrien sowie Probleme der Organisierung des tertiären Sektors (Dribbusch 2003) führten zu Mitgliederverlusten der DGB-Gewerkschaften. Die daraus entstandenen Probleme förderten eine Reihe von Gewerkschaftszusammenschlüssen, durch die sich die Zahl der DGB-Gewerkschaften sukzessive auf acht verringerte. Am bedeutsamsten war die 2001 erfolgte Bildung der Vereinten Dienstleistungsgewerkschaft (ver.di). Durch die Beteiligung der DAG und deren Aufgehen im DGB wurde das bis dahin wichtigste zwischengewerkschaftliche Konkurrenzverhältnis beendet. Im öffentlichen Dienst blieb zunächst die Konkurrenz zwischen den DGB-Gewerkschaften und dem Beamtenbund bestehen. Doch wurde auch hier 2006 eine tarifpolitische Kooperation eingeleitet, die 2008 formell vereinbart wurde.

Im Bahnbereich schlossen 2005 Transport, Service, Netze (TRANSNET) und die zum Beamtenbund gehörende Verkehrsgewerkschaft Gewerkschaft Deutscher Bundesbahnbeamten und Anwärter (GDBA) 
eine dreijährige Kooperationsphase mit der Bildung einer förmlichen Tarifgemeinschaft ab. Eine Entwicklung, die von der dritten Gewerkschaft in der Branche, der Gewerkschaft Deutscher Lokomotivführer (GDL), abgelehnt wurde.

\section{ENTWICKLUNG DER UNTERBIETUNGSKONKURRENZ}

Die Unterbietungskonkurrenz, die zuvor auf relativ kleine Nischen beschränkt war, nahm in den 1990er Jahren neue Dimensionen an. Hierbei spielte das Elektrohandwerk eine Vorreiterrolle. Bereits 1988 war der Norddeutsche Fachverband Elektrotechnik (NFE) aus den Tarifverhandlungen mit der IG Metall ausgestiegen und hatte mit der Christlichen Gewerkschaft Metall (CGM) einen ersten (Unterbietungs-)Tarifvertrag geschlossen, dessen praktische Bedeutung aber zunächst gering blieb. Nach dem Beitritt der DDR wurde diese Unterbietungspolitik jedoch nach und nach ausgeweitet. Im Ergebnis verdrängten die CGM-Tarifverträge in allen ostdeutschen und einer Reihe westdeutscher Tarifgebiete die IG-Metall-Flächentarifverträge. Ähnliche Entwicklungen gab es in anderen Handwerksbranchen, so insbesondere ab 2000 auch im Tischlerhandwerk (Buchholz 2006).

Ein weiterer Schwerpunkt wurde das privatisierte Gesundheitswesen, wo ein politisch gewollter Verdrängungswettbewerb zu erheblichem Druck auf tradierte Tarifstrukturen führte. So wechselte 2006 beispielsweise der Bundesverband Deutscher Privatkliniken (BDPK) nach dem Scheitern von Verhandlungen mit ver.di zur CGB-Gewerkschaft DHV (DHV - Die Berufsgewerkschaft), mit der dann das vom BDPK gewünschte Tarifergebnis möglich war.

Eine der folgenreichsten Unterbietungskonkurrenzen entwickelte sich im Bereich der Leiharbeit (Wiedemuth/Dombre 2008). Noch während die DGB-Gewerkschaften Anfang 2003 mit den beiden größten Arbeitgeberverbänden in der Leiharbeit, dem Bundesverband Zeitarbeit (BZA) und dem Interessenverband Deutscher Zeitarbeitsunternehmen (iGZ), verhandelten, wurde ein von der Tarifgemeinschaft Christlicher Gewerkschaften für Zeitarbeit und Personal Service Agenturen (PSA) (CGZP) mit einem kleinen nordbayerischen Arbeitgeberverband vereinbarter Abschluss bekannt. Dieser bewirkte, dass die zum gleichen Zeitpunkt noch nicht unterzeichneten Tarifverträge des DGB auf Druck der Arbeitgeberverbände nach unten korrigiert wurden, um überhaupt einen Abschluss sicherzustellen.

\section{NEUES PHÄNOMEN ÜBERBIETUNGSKONKURRENZ}

Im Gegensatz zur Unterbietungskonkurrenz, die in den meisten Fällen kaum Beachtung findet, stand die sich nach 2001 herausbildende Überbietungskonkurrenz sofort im Scheinwerferlicht. Vorreiter war die Vereinigung Cockpit (VC), ein 1969 gegründeter Zusammenschluss von Pilotinnen und Piloten. Diese hatte 1999, als die Beteiligung der DAG an der ver.di-Fusion absehbar wurde, ihre seit 1973 bestehende Tarifgemeinschaft mit der DAG gekündigt und bei der Lufthansa ihre tarifpolitische Eigenständigkeit durchgesetzt. 2001 verweigerte die VC dann dort die Übertragung der von DAG und ÖTV abgeschlossenen Tariferhöhung von 3,5\% und setzte mit mehreren spektakulären Streikaktionen erhebliche zweistellige Gehaltsverbesserungen durch (Bispinck/WSI-Tarifarchiv 2001; Schroeder/Greef 2008).

Relativ unbeachtet, weil sie ohne spektakulären Arbeitskampf auskam, verlief die tarifpolitische Verselbstständigung der Gewerkschaft der Flugsicherung (GdF). Diese war 2003 aus dem Verband deutscher Flugleiter (VdF) und dem Verband Deutscher Flugsicherungstechniker und -ingenieure hervorgegangen. Der VdF hatte bis 2002 mit ver.di eine nach der Privatisierung der Flugsicherung im Jahr 1993 zunächst mit der DAG geschlossene Kooperationsvereinbarung. Die GdF ist nun bei der Deutschen Flugsicherung die einzige Tarifpartei auf der Gewerkschaftsseite.

Der Marburger Bund (MB) setzte seine tarifpolitische Zusammenarbeit mit den DGB-Gewerkschaften bis 2005 fort. Der Bruch kam, als mit der Ablösung des alten Bundes-Angestelltentarifvertrages (BAT) durch einen neuen Tarifvertrag für den öffentlichen Dienst (TVöD) aus Sicht des MB massive Verschlechterungen für Mediziner drohten. Vor dem Hintergrund der durch die Privatisierungspolitik vorangetriebenen Umstrukturierung des öffentlichen Gesundheitswesens drängten innerhalb des Verbandes nicht zuletzt jüngere Assistenzärztinnen und -ärzte die Standesorganisation $\mathrm{zu}$ mehr gewerkschaftlichem Profil (Martens 2007; Greef 2008).
Bei der Bahn waren wie bei der Lufthansa und im Gesundheitswesen Privatisierung und nachfolgende Umstrukturierungen Auslöser dafür, dass sich die verschiedenen traditionellen Bahngewerkschaften neu orientieren mussten (Müller/ Wilke 2006). Während sich TRANSNET und GDBA aufeinander zu bewegten, setzte die GDL auf Eigenständigkeit und reklamierte ab 2002 die tarifliche Zuständigkeit für das gesamte Fahrpersonal. Der Konflikt eskalierte in der Tarifrunde 2007. Anfang 2008 konnte die GDL nach längeren von Streiks begleiteten Auseinandersetzungen ihre tarifpolitische Eigenständigkeit durchsetzen, wenn auch nur für die Lokführer (vgl. Hoffmann/Schmidt 2008).

Die 1992 gegründete Unabhängige Flugbegleiter Organisation (UFO) hatte inspiriert vom Schritt der VC ebenfalls bereits 1999 ihre Satzung so geändert, dass sie als Gewerkschaft auftreten konnte. Sie wurde bei der Lufthansa im Juli 2002 neben ver.di als gleichberechtigte Tarifpartnerin anerkannt. Es alleine besser zu machen, blieb jedoch zunächst nur Anspruch. UFO enttäuschte die Erwartungen vieler Mitglieder durch tarifvertragliche Konzessionen im Kontext der konzernweiten Restrukturierungspolitik der Lufthansa. ${ }^{1}$ Dergestalt unter Druck kündigte UFO anlässlich der ver.di-Tarifrunde im Sommer 2008 bei Lufthansa an, das von ver.di nach dem Arbeitskampf erzielte Ergebnis deutlich übertreffen zu wollen, wenn die eigenen Tarifverträge zum Jahresende 2008 ausliefen. UFO stand damit Anfang 2009 vor der Situation, eine tarifliche Überbietung erstmals auch tatsächlich durchsetzen zu müssen.

Von den genannten Gewerkschaften ist lediglich UFO eine echte Neugründung. VC, MB und GDL kommen aus einer berufsständischen und eher wirtschaftsfriedlichen Tradition. Die von ihnen geführten Streiks sind Ausdruck einer Vergewerkschaftlichung dieser Berufsorganisationen (Zeuner 2007). Dies bedeutet jedoch kein Einschwenken auf eine dauerhaft konfliktorientierte Tarifpolitik, wie sich bei allen dreien in den auf die Durchsetzung ihrer tariflichen Eigenständigkeit folgenden Tarifrunden zeigte.

\footnotetext{
1 Begleitet wurde diese Periode von inneren Querelen und Mitgliederverlusten (vgl. Hiekel 2008).
} 


\subsection{VERBREITUNG DER ÜBERBIETUNG}

Berufs- und Spartengewerkschaften sind nicht per se durchsetzungsfähiger als die Industriegewerkschaften. Bei den eigenständigen Tarifverträgen des Verbands medizinischer Fachberufe (VMF), ${ }^{4}$ der vor allem Arzthelferinnen organisiert, oder der Gewerkschaft ADEXA, die Apothekenbeschäftigte vertritt, kann ebenso wenig wie bei den verschiedenen Gewerkschaften im Kulturbereich von tariflicher Überbietung gesprochen werden. Sie agieren zumeist in gewerkschaftlichen Nischen und sind ganz überwiegend durchsetzungsschwach.

Die erfolgreiche Überbietungskonkurrenz ist auf sehr wenige Bereiche beschränkt. Von der Zahl der Beschäftigten her sind die Tarifverträge des Marburger Bundes für die Medizinerinnen und Mediziner am bedeutendsten. ${ }^{5}$ Die Tarifverträge von GdF, GDL sowie von VC betreffen hingegen relativ kleine Beschäftigtengruppen. In der Gesamtschau zeigt sich, dass tarifliche Überbietung an Voraussetzungen geknüpft ist, die nur von wenigen Gewerkschaften erfüllt werden und eine umfassende Ausweitung dieser Form tariflicher Konkurrenz unwahrscheinlich machen (u.a. Keller 2008). ${ }^{6}$

\subsection{VERBREITUNG DER UNTERBIETUNG}

In der Industrie ist es den christlichen Gewerkschaften bisher nicht gelungen, tarifpolitisch Fuß zu fassen. ${ }^{7}$ Zwar hat die Christliche Gewerkschaft Metall (CGM) ihre größten Mitgliedergruppen in einzelnen Betrieben der (westdeutschen) Metallindustrie, wo sie bei Betriebsratswahlen vereinzelt auch Mandate gewinnt, doch bleibt hier ihre Tariftätigkeit auf Anschlusstarifverträge beschränkt. Bei der CGM handelt es sich deshalb um den merkwürdigen Fall, dass eine Gewerkschaft nicht nur in einzelnen Ausnahmefällen, sondern systematisch gerade dort originär tariflich tätig wird, wo sie selbst, wenn überhaupt, nur marginal vertreten ist.

CGM-Tarifverträge dominieren, wie erwähnt, im Elektrohandwerk. Konkurrierende CGM-Flächentarifverträge mit unterschiedlicher Reichweite finden sich unter anderem im metallverarbeitenden Handwerk, im Heizungs- und Sanitärbereich sowie seit 2008 auch im Kfz-Gewerbe Nordrhein-Westfalens. Im Tischlerhand- werk haben 2008 von CGB-Gewerkschaften unterzeichnete Tarifverträge mit Ausnahme zweier Bundesländer die IG MetallTarifverträge abgelöst.

Im Dienstleistungsbereich wurden und werden vor allem mit der Gewerkschaft Öffentlicher Dienst und Dienstleistungen (GÖD) und der DHV Unterbietungstarifverträge geschlossen. CGB-Flächentarifverträge gibt es hier unter anderem im privaten Bewachungsgewerbe, bei den Privatkliniken sowie teilweise bei Großwäschereien. Haustarifverträge der DHV sowie von medsonet finden sich in Einrichtungen des Deutschen Roten Kreuzes sowie der Arbeiterwohlfahrt oder der Altenpflege. Die DHV ist teilweise auch im Finanzdienstleistungsbereich aktiv, wo auch der nicht dachverbandsgebundene Deutsche Bankangestellten Verband unterbietend in Erscheinung trat (Müller 2008).

Die quantitativ bedeutendste Unterbietungskonkurrenz findet in der Leiharbeit statt, wo neben den zwei DGB-Tarifverträgen mehrere von der CGZP abgeschlossene Verbandstarifverträge existieren, deren wichtigster mit dem Arbeitgeberverband Mittelständischer Personaldienstleister (AMP) geschlossen wurde. Die genaue Reichweite der einzelnen Tarifwerke lässt sich nicht feststellen. Allerdings kommt den zwischen CGZP und AMP geschlossenen Tarifverträgen mehr als nur marginale Bedeutung zu. ${ }^{8}$

Unterbietungstarifverträge sind somit deutlich weiter verbreitet als die ungleich

2 Alle in diesem Artikel genannten Mitgliederzahlen schließen nicht erwerbstätige Mitglieder ein und beruhen auf Gewerkschaftsangaben.

3 Das Urteil wurde am 10.2.2009 vom BAG bestätigt.

4 Bis 2006: Berufsverband der Arzt-, Zahnarzt- und Tierarzthelferinnen und -helfer.

5 Wobei durchaus strittig ist, wie weit das Volumen der vom $M B$ erreichten Abschlüsse tatsächlich die von ver.di abgeschlossenen Tarifregelungen übertrifft.

6 Auch wenn einzelne Spezialverbände, wie beispielsweise die 2002 gegründete Aircraft Release by Technicians and Engineers (A. R. T.E), die nach eigenen Angaben "Luftfahrzeug-Freigabe-Berechtigtes Personal und dessen Sonderinteressen " vertritt, diesen Schritt erwägen.

7 Für das immer wieder als Beleg bemühte Tarifwerk Phönix des CGM für die ostdeutsche Metallindustrie (Lesch 2008b, S. 304) fehlt jeglicher Nachweis, wo es denn zur Anwendung kommt.

8 Der AMP gab bei seiner Gründung 2004 an, Firmen mit mehr als 100.000 Beschäftigten zu vertreten. Der BZA vertrat 2008 nach eigenen Angaben Firmen mit 210.000 Beschäftigten und der iGZ Firmen mit etwa 185.000 Beschäftigten. 
prominentere, aber auf wenige Einzelbeispiele beschränkte tarifliche Überbietung. Auch wenn es nicht möglich ist, exakt zu ermitteln, für wie viele Beschäftigte solche Tarifverträge tatsächlich Anwendung finden, so kann mit Blick auf Leiharbeit und Bewachung sowie aufgrund ihrer Verbreitung im Handwerk davon ausgegangen werden, dass deren Zahl beträchtlich ist.

\section{Gewerkschaftspolitische Konsequenzen}

Die Tarif- und Gewerkschaftslandschaft ist in Bewegung. Bei tendenziell rückläufiger Tarifbindung und wachsendem Druck auf Tarifstandards sind die Industriegewerkschaften des DGB in den vergangenen Jahren von zwei Seiten her mit tariflicher Konkurrenz konfrontiert. Dabei taucht eine Reihe tarif- und gewerkschaftspolitischer Problemstellungen auf, die im Folgenden kurz diskutiert werden sollen.

\subsection{WIDERSPRÜCHLICHE TARIFPOLITISCHE SIGNALE}

Die Abschlüsse von VC, Marburger Bund und GDL haben bisher keine nachweislich negativen tariflichen Folgen für andere Beschäftigtengruppen gehabt. Das tarifpolitische Signal, das von diesen Abschlüssen ausgeht, erschwert eher die Erosion von Tarifstandards nach unten.

Genau dies bewirkt die Unterbietungskonkurrenz. Das von den CGB-Gewerkschaften tariflich vereinbarte Niveau liegt beim Entgelt zumeist nachweisbar unter, die Arbeitszeit dagegen oft über vergleichbaren Regelungen in DGB-Tarifverträgen. Daneben werden oftmals weniger Urlaub, keine Zuschläge oder auch keine oder nur minimale Sonderzahlungen vereinbart. Aus Sicht der Unternehmen steht nicht zufällig die Sorge im Vordergrund, die Überbietung könne zu einem Aufschaukeln von Tarifforderungen und damit zu einer Erhöhung der Personalkosten führen, während in der Unterbietungskonkurrenz Chancen für einen die Konzessionsbereitschaft fördernden gewerkschaftlichen Wettbewerb gesehen werden (Lesch 2008).

\subsection{INKLUSIVE ODER EXKLUSIVE SOLIDARITÄT?}

In der innergewerkschaftlichen Diskussion um die Überbietungskonkurrenz spielt die Frage der Solidarität eine große Rolle. Gewerkschaften, so das Argument, seien gegründet, um Konkurrenz zwischen Beschäftigten auszuschließen oder doch zumindest zu vermindern. Während die Einheits- und Industriegewerkschaften des DGB sich einer umfassenden inklusiven Solidarität verpflichtet fühlten, betrieben die Spartengewerkschaften eine exklusive, ausschließlich auf die eigene Berufsgruppe gerichtete Tarifpolitik.

Kurz-ScherflZeuner (2001) haben demgegenüber darauf hingewiesen, dass gewerkschaftliche Solidarität stets inklusive wie exklusive Komponenten hatte. Kein Tarifvertrag gilt für alle, und die angestrebte Kontrolle des Arbeitsmarktes beinhaltete immer auch problematische Zutrittsbeschränkungen. Das gewerkschaftliche Leitbild einschließender Solidarität, in dem die Durchsetzungsstärkeren die Schwächeren mitnehmen, ist in erster Linie aus der praktischen Einsicht entstanden, dass nur wenige Beschäftigtengruppen alleine erfolgreich sein können. Hinzu kommt die Erfahrung, dass erreichte Standards bei Entgelt und Arbeitsbedingungen dann am besten gegen Unterbietung abgesichert sind, wenn sie so verallgemeinert durchgesetzt werden, dass sie der wirtschaftlichen Konkurrenz entzogen sind. Der Aufbau inklusiver Solidarität wird aber schwieriger, je mehr Beschäftigte durch Ausgliederungen organisatorisch voneinander getrennt und durch veränderte Formen der Unternehmenssteuerung in neuer Weise zueinander in Konkurrenz gesetzt werden. Noch problematischer wird es, wenn das Interesse der Unternehmen am Tarifvertrag nachlässt und angesichts eines großen Arbeitskräfteangebots jegliche Regulierung von Lohn- und Arbeitsbedingungen als Wettbewerbshindernis betrachtet wird. In erster Linie sind es nicht Berufs- oder Spartengewerkschaften, sondern interessierte Unternehmen und öffentliche Arbeitgeber, die systematisch Flächentarife zerlegen und Tarifeinheiten zerbrechen.

In solchen Situationen, in denen es tarifpolitisch immer weniger Spielraum gibt, gewinnen exklusive Solidaritäten an Gewicht. Absenkungstarifverträge oder Standortvereinbarungen, in denen Besitzstände gesichert und zugleich schlechtere Kondi- tionen für Neueingestellte vereinbart werden, illustrieren, was gemeint ist (Zeuner 2007). Dies ist unter dem Gesichtspunkt der inklusiven Solidarität nicht wünschenswert, es ist aber nichts Ungewöhnliches und kaum durch moralische Appelle zu ändern.

In dieses Muster reiht sich auch das tarifpolitische Verhalten einzelner Spartengewerkschaften ein, die den „Großgewerkschaften" Politik zulasten der von ihnen vertretenen Funktionseliten vorwerfen. So meint das bei VC für Tarifpolitik zuständige Vorstandsmitglied Michael Tarp: „Die Solidarisierungsbemühungen der Großgewerkschaften haben in der Luftfahrtbranche eine Tarifpolitik zur Folge gehabt, die sich am schwächsten Glied in der Kette orientiert hat" (2008, S. 403). Und nach Auffassung des Hauptgeschäftsführers des Marburger Bundes Armin Ehl hätten die Ärztinnen und Ärzte „die von den Großgewerkschaften beschworene, Solidarität' der verschiedenen Beschäftigtengruppen seit Langem nicht mehr [gespürt]", denn die Tarifverhandlungen über BAT und TVöD hätten dazu geführt, ,dass die unteren Vergütungsgruppen zulasten der höheren besser gestellt wurden“ (Ehl 2008, S. 406).

Diese Argumentation unterschlägt, dass es in der Vergangenheit gerade die vermeintlich schwächeren Glieder in der Kette sowie „die unteren Vergütungsgruppen“ waren, die die Tarifsysteme aufgebaut und gesichert hatten, auf denen sowohl VC wie MB nun aufbauen. Es waren nicht Ärzte, sondern Beschäftigte der Müllabfuhr, die, wenn nötig, im öffentlichen Dienst gestreikt haben. Weder Piloten noch Ärzten ist vorzuwerfen, dass sie sich für ihre Interessen einsetzen, doch dass sie dabei alle anderen aus dem Blick verlieren, unterstreicht ihr exklusives Solidaritätsverständnis. Dass 2008 im Aufsichtsrat der Lufthansa der als Gegenkandidat zum ver.di-Vorsitzenden Frank Bsirske angetretene Bewerber der VC bei der Wahl zum stellvertretenden Vorsitzenden keine Chance hatte, obwohl ver.di nach einer herben Wahlniederlage nur noch drei Mandate von zehn auf der Arbeitnehmerseite stellte, verdankte sich offenbar nicht zuletzt der über ver.di hinausreichenden Ablehnung einer als elitär begriffenen Interessenpolitik der VC (Hiekel 2008). Wer sich wie der MB zur „Vertretung der qualifiziertesten Arbeitnehmergruppe in den Krankenhäusern" (Ehl 2008) erklärt und eine Umverteilung zugunsten der unteren Vergütungsgruppen beklagt, 
verdrängt, dass gerade Krankenhausarbeit Teamarbeit bedeutet und provoziert Spaltungen und Entsolidarisierungen.

Umgekehrt ist der seitens der DGB-Gewerkschaften gelegentlich erhobene Vorwurf, die tarifpolitischen Alleingänge der Spartengewerkschaften gingen zulasten anderer Beschäftigter, nicht hilfreich. Erstens sind, worauf Detlef Hensche hingewiesen hat (2007), Verteilungskonflikte innerhalb der Klasse nichts prinzipiell Ungewöhnliches. Sie sind Bestandteil der Tarifpolitik und Gegenstand innergewerkschaftlicher Entscheidungsfindung. Verwiesen sei nur auf die alte Debatte um Festbeträge oder Prozentforderungen. Zweitens sind die Verteilungsspielräume zwischen den Klassen nicht starr, sondern selbst Ergebnis tariflicher Auseinandersetzung beziehungsweise politischen Streits (Zeuner 2007). Statt des gegenseitigen Vorwurfs der Umverteilung zulasten der jeweils eigenen Mitgliedschaft erscheint es unter dem Aspekt des Wiederaufbaus inklusiver Solidarität sinnvoller, gemeinsam die Verteilungsmenge zu erhöhen. In diese Richtung weist beispielsweise die von ver.di gemeinsam mit $\mathrm{MB}$ und dbb geführte Kampagne „Der Deckel muss weg“, mit der eine Gesundheitspolitik attackiert wird, die letztlich allen Gewerkschaften die tarifpolitischen Grundlagen entzieht.

\subsection{DIE PROBLEMATIK DER UNTERBIETUNG}

\section{KEINE GRUNDLAGE FÜR KOOPERATION}

Im Gegensatz zur Überbietungskonkurrenz ist ein kooperatives Arrangement der DGB-Gewerkschaften mit der unterbietenden Tarifpolitik der CGB-Gewerkschaften kaum vorstellbar. Es kann nicht verwundern, dass die DGB-Gewerkschaften diese Tarifpolitik mit allen gewerkschaftlichen und juristischen Mitteln bekämpfen. Der juristische Weg, der darauf zielt, den unterbietenden Gewerkschaften die Tariffähigkeit abzuerkennen, war jedoch nur begrenzt erfolgreich und seine zukünftigen Erfolgsaussichten sind ungewiss (Bispinck/ Dribbusch 2008).

In einzelnen Fällen gelingt es DGB-Gewerkschaften, durch die Mobilisierung der von Verschlechterungen bedrohten Beschäftigten die Anwendung von CGBTarifverträgen zu verhindern beziehungsweise wieder zurückzudrängen (Bispinck/
Dribbusch 2008). Oft entwickelt sich in diesen Branchen eine gespaltene Tariflandschaft. Die Richtung ist eindeutig: Gut organisierte Belegschaften, vor allem in den größeren Betrieben, behalten DGB-Tarifverträge; in den anderen Betrieben wird es keine oder allenfalls „christliche“ Tarifverträge geben. So konnte beispielsweise die IG-Metall 2008 nach entsprechenden Auseinandersetzungen im Kfz-Handwerk Nordrhein-Westfalens in fast allen Betrieben mit Betriebsräten die IG-Metall-Tarifverträge halten oder zurückgewinnen. Ähnliches gelang ver.di bei großen Privatkliniken. In der Fläche ist solche Durchsetzungsfähigkeit in kleinbetrieblich organisierten Branchen aber nur sehr schwer herstellbar.

\section{POLITISCHE AUFWERTUNG DER CGB-GEWERKSCHAFTEN}

In den letzten Jahren lässt sich im Zusammenhang mit der Diskussion um gesetzliche Mindestlöhne eine politische Aufwertung der CGB-Gewerkschaften beobachten. Dies zeigt sich beispielsweise in der Vehemenz, mit der die CDU/CSU die Unterbietungstarifverträge des CGB in der Leiharbeit verteidigt. Nachdem 2007 von der großen Koalition beschlossenen wurde, in ausgewählten Branchen tarifliche Mindestlöhne über das Arbeitnehmer-Entsendegesetz allgemeinverbindlich erklären zu lassen, wurde 2009 die Leiharbeit nicht darin aufgenommen. Hintergrund ist eine entsprechenden Lobbyarbeit des CGB und die Weigerung des Arbeitgeberverbandes AMP, die mit der CGZP geschlossenen unterbietenden Tarifverträge zugunsten eines einheitlichen Mindestlohntarifvertrages aufzugeben.

In anderen Branchen, die mit dem Ziel von Mindestlohnregelungen in das Entsendegesetz aufgenommen wurden, stellten Unterbietungskonkurrenzen die betroffenen DGB-Gewerkschaften vor schwierige Entscheidungen. Im Bereich der Textilen Dienste (Großwäschereien) gibt es zwei konkurrierende Arbeitgeberverbände, von denen einer mit der IG Metall und ein anderer, kleinerer mit der DHV Flächentarifverträge abgeschlossen hatte. Anfang 2009 wurde nun ein in textgleichen Versionen von allen Beteiligten unterzeichneter Mindestentgelttarifvertrag vereinbart, dessen Niveau zwar oberhalb des gegenwärtigen DHV-Tarifs, aber unterhalb des IG MetallTarifvertrages liegt. Aus Sicht der IG Metall, die vor einer schwierigen Abwägung stand, steht dabei der Vorteil der Allgemeinverbindlichkeit, durch die vor allem die nicht tarifgebundenen Unternehmen unter Druck geraten, die bisher selbst unter DHV-Tarif bezahlen, dem Nachteil gegenüber, dass die Lohnkonkurrenz nicht beendet werden konnte. ${ }^{9}$ Zugleich wurde DHV durch ihre Beteiligung am Mindestlohntarifvertrag aufgewertet.

Einen Prestigegewinn konnte im gleichen Kontext auch die GÖD verbuchen, die sogar als alleinige Gewerkschaft im privaten Bewachungsgewerbe im Juni 2008 mit dem Bundesverband Deutscher Wach- und Sicherheitsunternehmen (BDWS) einen Mindestlohntarifvertrag abgeschlossen hat. Zuvor waren Verhandlungen zwischen BDWS und ver.di gescheitert. Grund für das Scheitern war die Weigerung des BDWS, einem tariflichen Stufenplan zuzustimmen, der in absehbarer Zeit die untersten Löhne in der Branche auf 7,50 € angehoben hätte. Angesichts ihrer Mindestlohnkampagne „Kein Lohn unter 7,50€ pro Stunde" befand sich ver.di in dem Dilemma, entweder weiter mit dem Vorwurf konfrontiert zu werden, sie tarifiere selbst Niedriglöhne, oder aber diesen Widerspruch in Kauf zu nehmen, um der GÖD nicht alleine das Feld zu überlassen.

Die Frage, ob mangels branchenweiter Durchsetzungsfähigkeit ein Rückzug aus der Fläche zugunsten tariflicher Insellösungen in gut organisierten Betrieben die gewerkschaftspolitisch bessere (Zwischen)Lösung darstellt, wird die Gewerkschaften weiter beschäftigen.

\section{Ausblick}

Die Tariflandschaft in Deutschland befindet sich in einem Dezentralisierungs- und Ausdifferenzierungsprozess, dessen Dynamik aus wirtschaftlichen und politischen Entscheidungen resultiert und in dem zwischengewerkschaftliche Konkurrenzen einen Teilaspekt bilden. Während die Unterbietungskonkurrenz ohne aktives Zutun

\footnotetext{
Diese findet weiter statt zwischen den vor allem kleineren Betrieben, die sich zukünftig am Mindestlohntarifvertrag orientieren werden, und den vor allem größeren Unternehmen, die weiter durch den IG Metall-Flächentarifvertrag gebunden sind
} 
interessierter Unternehmen weder Gegenwart noch Zukunft hätte, resultiert die Überbietungskonkurrenz aus originär gewerkschaftlichem Handeln, dass durch hohe Organisations- und Mobilisierungsbereitschaft der betreffenden Beschäftigten gekennzeichnet ist. Was die zwischengewerkschaftlichen Perspektiven angeht, so erscheint im Falle dieser Konkurrenzen eine neu begründete tarif- und gewerkschaftspolitische Zusammenarbeit zwischen DGB- und Berufsgewerkschaften, für die es ja bereits Beispiele gibt, nicht prinzipiell ausgeschlossen.

Die Unterbietungskonkurrenz ist dagegen im Kern eine gegen die DGB-Gewerkschaften gerichtete Tarifpolitik zulasten der Beschäftigten. Leiharbeit, Textilreinigung und Bewachung sind nur die auffälligsten Beispiele dafür, dass sich Unterbietungskonkurrenz zunehmend etabliert und po- litisch akzeptiert wird. Ideologisch flankiert wird diese Entwicklung von Wirtschaftswissenschaftlern, die den infolge der Deregulierung des staatlichen Sektors und der Leiharbeit eröffneten Unterbietungswettbewerb als Aufbrechen von Tarifkartellen und Entstehung „neuer Märkte für Gewerkschaftsleistungen“ (Wey 2008) begrüßen. Während von dieser Seite gerne auf fallende Mitgliederzahlen der DGBGewerkschaften verwiesen wird, wird die fehlende Repräsentativität der Unterbietungsgewerkschaften wohlweislich nicht thematisiert.

Die Unternehmerseite argumentiert in der Frage Tarifkonkurrenz und Tarifeinheit widersprüchlich. Einerseits treibt sie die Differenzierung der Tariflandschaft und der Tarifstandards auf Branchen- und Betriebsebene auf vielfältige Weise voran und nutzt dabei, wo zweckdienlich, auch beste- hende zwischengewerkschaftliche Konkurrenzen. Zum andern fordert sie dort, wo Überbietungskonkurrenz droht, die Durchsetzung des Prinzips der Tarifeinheit auf betrieblicher Ebene notfalls auch durch gesetzliche Maßnahmen abzusichern (Hundt 2007; Lesch 2008). Dahinter steckt der strategische Ansatz einer Tarifpolitik, die inhaltlich auf eine Deckelung, Differenzierung und Variabilisierung der Tarifstandards zielt und ordnungspolitisch von der betriebsbezogenen Tarifeinheit und der branchenbezogenen Friedenspflicht profitieren will. Inwieweit die Unternehmen mit diesem Konzept erfolgreich sein werden, hängt nicht zuletzt davon $\mathrm{ab}$, ob es den DGB-Gewerkschaften gelingt, wirksame Gegenstrategien zu entwerfen und entsprechende Verhandlungsmacht aufzubauen.

\section{LITERATUR}

Bayer, H. (1980): Zur gewerkschaftlichen Organisation der Angestellten im privaten Dienstleistungssektor. Organisationsstrukturelle Wandlungen der DGB-Gewerkschaft Handel, Banken und Versicherungen in Konkurrenz zur Deutschen Angestelltengewerkschaft 1950-1975, Frankfurt/Main Bispinck, R./WSI-Tarifarchiv (2001): Tarifpolitischer Halbjahresbericht, Düsseldorf

Bispinck, R. (2006): Abschied vom Flächentarifvertrag? Der Umbruch in der deutschen Tariflandschaft, in: WSI (Hrsg.): Tarifhandbuch 2006, Köln, S. 41-66

Bispinck, R./Dribbusch, H. (2008): Tarifkonkurrenz der Gewerkschaften zwischen Über- und Unterbietung, in: Sozialer Fortschritt 6, S. 153-163

Buchholz, G. (2006): Sie können nur billig. "Christliche“ Gewerkschaften und wie sie Arbeitnehmerinteressen verkaufen, Heidelberg

Dribbusch, H. (2003): Gewerkschaftliche Mitgliedergewinnung im Dienstleistungssektor, Berlin

Dribbusch, H./Schulten, T. (2007): The End of an Era: Structural Changes in German Public Sector Collective Bargaining, in: Leisink, P./Steijn, B./Veersma, U. (Hrsg.): Industrial Relations in the New Europe. Enlargement, Integration and Reform, Cheltenham, S. 155-176
Greef, S. (2008): Die deutsche Gewerkschaftslandschaft im Wandel. Neue Gewerkschaftskonkurrenz am Beispiel des Krankenhaussektors, Arbeitspapier zur Tagung der Hans-Böckler-Stiftung „Politik zur Erneuerung der Gewerkschaften“, 1.-2. September, Kassel

Hensche, D. (2007): Lokführer als Avantgarde?, in: Blätter für deutsche und internationale Politik 9, S. 1029-1032

Hiekel, D. (2008): Aufsichtsrat - in eigener Sache. (Statement im Vorfeld der Personalvertretungswahlen bei Lufthansa 2008) online unter: http://www.pv-wahl2008.de/dhar2.html (11.02.2009)

Hoffmann, J./Schmidt, R. (2008): Der Streik der Lokführer-Gewerkschaft GDL - Anfang vom Ende des deutschen Systems der industriellen Beziehungen?, in: Prokla 151, S. 323-342

Hümmerich, K./Holthausen, J. (2006): Soziale Mächtigkeit durch aktive Teilnahme am Tarifgeschehen. Der CGM-Beschluss des 1. Senats, in: Neue Zeitung für Arbeitsrecht (NZA) 19, S. 1070-1078

Hundt, D. (2007): „Arbeitgeberpräsident Dr. Dieter Hundt unterstützt die Vorschläge des Bahn-Vorstandsvorsitzenden Mehdorn für einen Ordnungsrahmen zur Sicherung der Tarifeinheit" ${ }^{\prime}$, Presse-Information 97 vom 14.11. 
Keller, B. (1993): Arbeitspolitik des öffentlichen Sektors, Baden-Baden Keller, B. (2008): Der aufhaltsame Aufstieg von Berufsverbänden,

in: Sozialer Fortschritt 6, S. 163-173

Kurz-Scherf, I./Zeuner, B. (2001): Politische Perspektiven der Gewerkschaften zwischen Opposition und Kooperation, in: Gewerkschaftliche Monatshefte 3, S. 147-160

Lesch, H. (2008): Spartengewerkschaften - Droht eine Destabilisierung des Flächentarifvertrages?, in: Sozialer Fortschritt 6, S. 144-153

Martens, H. (2007): Primäre Arbeitspolitik und neue Gewerkschaft?

Der Ärztestreik im Frühjahr und Sommer 2006, in: Jahrbuch für Kritische Medizin 44, S. 120-137

Müller, H.-P./Wilke, M. (2006): Gestaltend Einfluss nehmen, Bahngewerkschaft und Bahnreform 1993-2005, Berlin

Müller, M. (2008): Verband der Erfüllungsgehilfen, in: Mitbestimmung 4, S. 36-37
Schroeder, W. (2008): Die Konkurrenten der Einheitsgewerkschaft. Wie sich das deutsche Modell verändert, in: Mitbestimmung 4, S. 10-15 Schroeder, W./Greef, S. (2008): Industrie und Spartengewerkschaften im Konflikt. Organisatorische Voraussetzungen und realisierte Gelegenheitsstrukturen, in: Industrielle Beziehungen 4, S. 329-355

Silver, B. (2005): Forces of Labor. Arbeiterbewegungen und Globalisierung seit 1870, Berlin

Wey, C. (2008): Neue Mindestlohngesetze vernichten Gewerkschaftskonkurrenz, in: DIW-Wochenbericht 47, S. 750

Wiedemuth, J./Dombre, R. (2008): Regulierung der Zeitarbeit durch Tarifpolitik. Eine Zwischenbilanz, in: Bispinck, R. (Hrsg.): Verteilungskämpfe und Modernisierung. Aktuelle Entwicklungen in der Tarifpolitik, Hamburg, S. 159-181

Wilhelm, G./Dannhorn, W. (2006): CGM tariffähig. Neue Möglichkeiten für Arbeitgeber?, in: Arbeit und Arbeitsrecht 6, S. 343-345 